\title{
Parallel, exhaustive processing underlies logarithmic search functions: Visual search with cortical magnification
}

\author{
Zhiyuan Wang $^{1} \cdot$ Alejandro Lleras $^{1} \cdot$ Simona Buetti $^{1}$
}

Published online: 17 April 2018

(C) Psychonomic Society, Inc. 2018

\begin{abstract}
Our lab recently found evidence that efficient visual search (with a fixed target) is characterized by logarithmic Reaction Time (RT) $\times$ Set Size functions whose steepness is modulated by the similarity between target and distractors. To determine whether this pattern of results was based on low-level visual factors uncontrolled by previous experiments, we minimized the possibility of crowding effects in the display, compensated for the cortical magnification factor by magnifying search items based on their eccentricity, and compared search performance on such displays to performance on displays without magnification compensation. In both cases, the RT $\times$ Set Size functions were found to be logarithmic, and the modulation of the log slopes by targetdistractor similarity was replicated. Consistent with previous results in the literature, cortical magnification compensation eliminated most target eccentricity effects. We conclude that the log functions and their modulation by target-distractor similarity relations reflect a parallel exhaustive processing architecture for early vision.
\end{abstract}

Keywords Visual search $\cdot$ Cortical magnification $\cdot$ Eccentricity $\cdot$ Similarity

The visual-search paradigm has been widely used to study human visual attention. Most theories of visual search assume two stages of processing: an early, parallel stage and a later, serial stage (Desimone \& Duncan, 1995; Itti \& Koch, 2001; Theeuwes, 2010; Treisman \& Gelade, 1980; Wolfe, 1994). The first stage performs basic functions like extraction of simple features and segmentation of objects from the background. In the second stage, focused attention compares one or a few items at a time to a target template. In this literature, inferences are typically made based on differences in search slopes, which are slope coefficients of linear regressions on the Reaction Time (RT) $\times$ Set Size function. This approach implies the assumption that these RT $\times$ Set Size functions are inherently linear. When slope coefficients are found to be near zero, the usual conclusion is that searches were completed within the first parallel stage of processing, and they are referred to as efficient or pop-out searches (Lagroix, Di Lollo, \& Spalek, 2015; Treisman \& Gelade, 1980). Efficient search was

Zhiyuan Wang

zhiyuanwang42@gmail.com

1 Department of Psychology, University of Illinois, 603 E Daniel St, Champaign, IL, USA further assumed to be completed preattentively, implying that attentive selection in the parallel processing stage acts like a passive filter. While the practice of only analyzing RT $\times$ Set Size function with a linear model has been questioned from various perspectives (e.g., Carrasco, Evert, Chang, \& Katz, 1995; Townsend, 1990), the notion that parallel processing being preattentive still had a long-term influence on the field.

A recent investigation by Buetti, Cronin, Madison, Wang, and Lleras (2016; see also Wang, Buetti, \& Lleras, 2017) reported that, in efficient search tasks with fixed targets, the RT $\times$ Set Size function is in fact not linear, but logarithmic. This result further challenges the continued practice of always treating RT $\times$ Set Size functions as linear (e.g., Wolfe \& Horowitz, 2017). More importantly, the authors found evidence for a systematic modulation of these logarithmic slopes by target-distractor similarity, indicating modulations by task goals in the early stage of visual search. The observed logarithmic slopes were shallower for distractors less similar to the target and steeper for distractors more similar to the target. Note that these distractors were still sufficiently dissimilar from the target to be rejected in parallel during Stage 1 processing. Buetti and colleagues referred to these distractors as lures and contrasted them with candidates, which are distractors that are sufficiently similar to the target to require focused attention to determine their identity with a high degree 
of confidence (see also Lleras, Buetti \& Mordkoff, 2013; Neider \& Zelinsky, 2008). Inspired by Townsend and Ashby's (1983) seminal work, Buetti et al. (2016) interpreted their findings as evidence that early visual processing has a parallel architecture and an exhaustive termination rule, and that processing of each item can be modeled as noisy accumulators whose efficiency depends on that item's similarity to the target. The end result of this parallel processing stage is the rejection of lure items as potential targets, while candidates are passed on to a second serial stage for further inspection. Thus, the rejection of lures and the inspection of candidates in the process of finding a fixed target are associated with distinct behavioral signatures: The former results in logarithmic RT $\times$ Set Size functions, the latter produces linear RT $\times$ Set Size functions. Wang et al. (2017) replicated the same results using images of real-world objects, and, more importantly, working with the theory proposed by Buetti et al. (2016), were able to successfully predict search performance on lure-heterogeneous displays based on performance observed in homogeneous displays. Together, these findings highlight that early processing in visual search is nontrivial: It has a parallel, exhaustive mechanism as revealed by the logarithmic RT $\times$ Set Size functions, and its processing efficiency is also modulated by targetdistractor similarity, which contradicts the view that early parallel processing passively filter out irrelevant features.

However, it remains unclear what is the contribution of low-level visual factors such as cortical magnification to the logarithmic nature of these efficient search functions. That is, given the displays used in Buetti et al., where stimuli were placed at various eccentricities, one may ask whether the logarithmicity observed in those search functions is intrinsic to fixed-target efficient search tasks or whether it is a result of the specific stimulus arrangement used in that study, and more specifically, of varying target eccentricity. Carrasco et al. (1995) reported eccentricity effects in conjunction searches where traditional search slopes were greater with increasing target eccentricity. Later, Carrasco and Frieder (1997) demonstrated that such eccentricity effects can be neutralized by cortical magnification manipulations (see also Carrasco, McLean, Katz, \& Frieder, 1998). Cortical magnification refers to the fact that different parts of the retina are disproportionally represented in the visual cortices: The size of receptive fields for visual cortex neurons increase with retinal eccentricity (Dow, Snyder, Vautin, \& Bauer, 1981), which means foveal regions are more densely represented by cortical neurons than peripheral regions (despite that the fovea covers only a small portion of the visual field). Consequently, the same retinal image of an object will be represented by fewer neurons if it falls in the periphery than if it falls in the fovea. These factors can lead to decreased performance in many visual tasks as stimulus eccentricity increases (for a review, see Strasburger, Rentschler, \& Jüttner, 2011), and thus potential reaction-time differences in visual search. Both in Buetti et al. (2016) and in
Wang et al. (2017), we took the conventional approach of averaging across target eccentricity conditions and did not explicitly account for effect of target eccentricity or cortical magnification. In fact, when we plotted RTs as a function of position in the display (Buetti et al., 2016, Appendix 2), RTs did seem to increase as target eccentricity increased in those displays. Thus, the previously observed logarithmic RT $\times$ Set Size functions could be attributed to target eccentricity effects instead of our proposed mechanism of parallel search processing.

The current study aims to test whether target eccentricity was responsible for the logarithmic RT $\times$ Set Size functions we observed previously, by manipulating eccentricity and cortical magnification in displays that minimize crowding. Specifically, we compared a condition where search stimuli were magnified according to Rovamo and Virsu's (1979) measured cortical magnification factor to another condition without such compensation. The goal of the former condition was to roughly compensate for the quantitative differences in cortical representation of stimuli at different eccentricities. Furthermore, we used Bouma's law to minimize crowding effects by designing a concentric display where the distance between items was always larger than the critical spacing of crowding (see the Method section for more details). According to our theory, we should observe logarithmic RT $\times$ Set Size functions modulated by target-lure similarity in both magnified and not magnified conditions. This follows because what we proposed as responsible for logarithmic RT functions are factors related to the processing architecture of fixed-target efficient search tasks: parallel, exhaustive processing, where information about each item accumulates noisily at each location until a decision threshold is reached. The logarithmicity, then, arises from the combination of the stochastic accumulation process and the exhaustive termination rule. In other words, it is largely independent of the efficiency with which individual items are processed, which eccentricity might modulate. In contrast, if log-like RT $\times$ Set Size functions are due to target eccentricity effect, then we should observe nonlogarithmic and perhaps linear functions under the cortically magnified condition, since the eccentricity effects should be eliminated.

\section{Method}

Participants Thirty-three subjects participated in exchange for course credit in a psychology class. We initially planned to collect data from 20 participants, which would have achieved about $90 \%$ power at $\alpha=0.01$ according to previously estimated size of the Set Size $\times$ Lure Type interaction effect. However, due to miscommunication with research assistants, more data were collected. Participants were screened for normal color vision using the Ishihara Color Test. No participants 
were excluded due to abnormal color vision or low visual acuity. Participants whose overall accuracy was below $92 \%$ were excluded (this accuracy criterion was chosen to be higher than the usual $90 \%$ since we have a surplus of data). For the 29 participants included in analyses, all were right-handed, 19 were female, and their age ranged from 18 to 22 years. This experiment was approved by the Institutional Review Board of the University of Illinois at Urbana-Champaign.

Apparatus and stimuli Stimuli were presented on a 20-inch CRT monitor. Participants sat in a dimly lit room at a viewing distance of $55 \mathrm{~cm}$, with their chin on a chin rest to ensure viewing position. The experiment was programmed in MATLAB with Psychtoolbox 3.0 (Kleiner et al., 2007).

A blue half disk was chosen as the search target, whose arc faces either leftward or rightward. Lures were either cyan circles (high similarity to the target) or orange diamonds (low similarity to the target). The positions of these search objects on the screen were determined on the basis of a grid consisting of three concentric circles, each containing 12 evenly spaced possible positions. The eccentricities of these circles were $4.5^{\circ}, 8.4^{\circ}$, and $15.4^{\circ}$ of visual angle. Search items were positioned so that the display would be symmetrical along the vertical midline. A small amount of random jitter was added to each item's final position. This specific concentric grid display was designed to minimize crowding effects based on Bouma's equation (Pelli, 2008). In trials without cortical magnification compensation, stimulus size was not varied (about $1 \times 1$ degrees of visual angle for lures and 0.5 $\times 1$ for the target). In trials with cortical magnification compensation, size of stimuli presented on the middle and outer circle of the grid were magnified. The magnification factor $\mathrm{M}$ followed Rovamo and Virsu (1979), who reported M as an approximately linear function of eccentricity (see Fig. 1 for examples). More specifically, a stimulus with an eccentricity of $\varphi$ was magnified (both horizontally and vertically) by $\mathrm{M}$ :

$M=\left(1+C \varphi+0.00007 \varphi^{3}\right) /\left(1+C \varphi_{0}+0.00007 \varphi_{0}^{3}\right)$,

where $\varphi_{0}$ is the smallest eccentricity $\left(4.5^{\circ}\right)$ in our display arrangement, and $\mathrm{C}$ is a constant that depends on the angular position $\theta$ of the stimulus. $C$ could take one of three values for different values of $\theta$ :

$\begin{array}{lll}C=0.32 \text { if } \theta=15^{\circ}, & 165^{\circ},-15^{\circ}, & \text { or }-165^{\circ} ; \\ C=0.36 \text { if } \theta=45^{\circ}, & 135^{\circ},-45^{\circ}, & \text { or }-135^{\circ} ; \\ C=0.40 \text { if } \theta=75^{\circ}, & 105^{\circ},-75^{\circ}, & \text { or }-105^{\circ} \text {. }\end{array}$

Procedure Instructions were both shown on the screen and delivered verbally to participants at the beginning of the experimental session. Participants were told to look for the blue half-disk target and to indicate whether the arc faced leftward or rightward. They were asked to press the left arrow key with their left index finger when the target's arc faced to the left, and the right arrow key with their right index finger when the arc faced to the right. To control for speed-accuracy trade-off, they were instructed to respond as soon as possible without making errors. Participants were also told about the search display appearing for only a short moment (see below), and were encouraged to keep fixating at the center of the screen when doing the search task.

Trials started with a central white fixation cross presented for $600 \mathrm{~ms}$. After an onset delay of $450 \mathrm{~ms}$, the search scene was displayed for $100 \mathrm{~ms}$. This short presentation duration was chosen to prevent eye movements during search, which was necessary to ensure the validity of the cortical magnification manipulation. Participants were also verbally instructed to search for the target without moving their eyes, although eye movements were not monitored. The trial terminated as soon as the participant pressed a response key. On trials where participants made an error, a warning tone $(750 \mathrm{~Hz}$ sine wave lasting $250 \mathrm{~ms}$ ) followed the participant's response. The intertrial interval varied randomly between 1.5 to 1.7 seconds. Each experiment session started with a practice block of 16 trials that contained both magnified and nonmagnified trials.

Design The independent variables are magnification (with or without cortical magnification compensation), lure type (orange diamond or cyan circle), and set size (2, 6, 16, or 32 search objects). In addition, a target-only condition was included, where the only item on the display was the target. All factors were fully crossed, so that there were a total of 18 conditions. There were 44 repetitions per condition, leading to a total 792 trials in an experiment session. The magnification factor was blocked while the other two factors were randomly intermixed. Ordering of the blocks was counterbalanced across subjects, and each block contained 36 trials. At the end of each block there were break periods that lasted up to 30 seconds, or until the participant chose to resume the experiment. There were on average 264 trials where the target was at $4.5^{\circ}, 8.4^{\circ}$, or $15.4^{\circ}$ degrees of eccentricity.

\section{Results}

Log versus linear fits comparison Only trials with correct responses were included for analysis in our reported results. First, we analyzed the mean RT $\times$ Set Size function to compare the logarithmic and linear fits. Across-subject mean RTs and standard errors of the mean were computed for each of the 18 conditions. These data are shown in Fig. 2. Table 1 shows the R-squared $\left(R^{2}\right)$ values and log likelihoods of the four regression models. The results indicate that the logarithmic model consistently fits the data better than the linear model, 

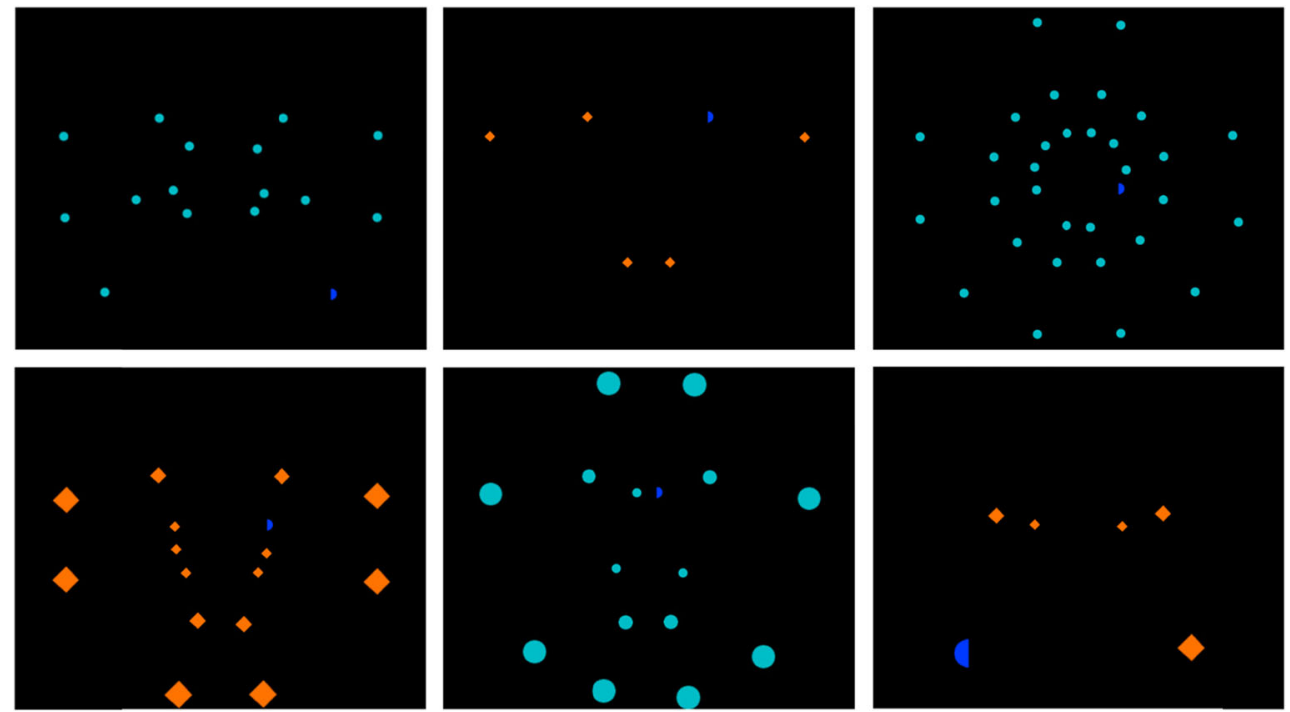

Fig. 1 Sample search displays. Participants were asked to search for the blue half-disc target among cyan circles (high similarity lures) or orange diamonds (low similarity lures). Top row: no compensation for cortical

magnification; bottom row: magnified displays to compensate for cortical magnification. (Color figure online)

with a relative likelihood ratio of 19.11 or more and $R^{2}$ of 0.95 or more. Across-subject mean error rates are plotted in Fig. 3. Combining Figs. 2 and 3, we observe no apparent speed-accuracy trade-off pattern.

Next, for each combination of magnification and lure type, we computed logarithmic and linear regressions on each subjects' data and computed the log likelihood values of both logarithmic and linear models. A two-sample $t$ test on these log likelihood values suggested that log likelihoods of logarithmic models are greater than those of linear models, $t(230)$ $=3.447, p<.001$, Hedge's $g=0.4512$.
ANOVA on slopes and intercepts To further understand how cortical magnification changes the processing in efficient visual search, we conducted analyses of variance on the two coefficients of the logarithmic RT $\times$ Set Size regression model as dependent variables. This approach allowed us to decompose RT variability into two components: the log slope coefficients reflecting the RT variability as a function of set size (i.e., a measure of set size effect) and the intercept coefficients representing RT variability that's unrelated to set size. Using magnification and lure type as within-subjects fixed effects, subject as a random effect, and the logarithmic slope

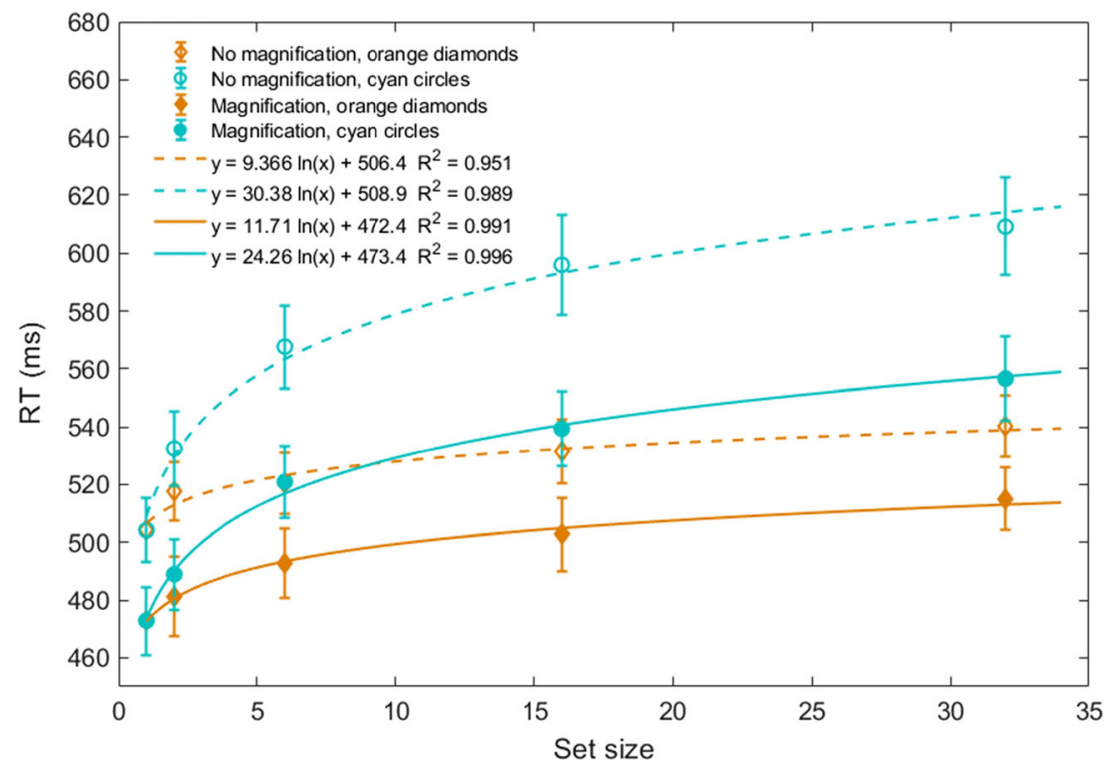

Fig. 2 Mean reaction time as a function of set size, grouped by magnification and lure type. Error bars indicate one standard error of mean. Curves are least-square best-fitting logarithmic functions. (Color figure online) 
Table 1 Comparison of logarithmic versus linear regression fits to RT $\times$ Set Size function for each of the four main conditions

\begin{tabular}{|c|c|c|c|c|c|}
\hline \multirow[b]{2}{*}{ Condition } & \multicolumn{2}{|l|}{$R^{2}$} & \multicolumn{3}{|c|}{ Log likelihood } \\
\hline & Log model & Linear model & Log model & Linear model & Likelihood Ratio \\
\hline No magnification, orange diamond & 0.951 & 0.840 & -12.11 & -15.06 & 19.11 \\
\hline No magnification, cyan circle & 0.989 & 0.763 & -14.05 & -21.82 & 2,368 \\
\hline Magnification, orange diamond & 0.991 & 0.892 & -8.88 & -15.09 & 497.7 \\
\hline Magnification, cyan circle & 0.996 & 0.813 & -10.66 & -20.09 & 12,457 \\
\hline
\end{tabular}

coefficients as dependent variables, a mixed-effect ANOVA revealed that slope coefficients were significantly larger for cyan circles (28.1) than for orange diamonds $(12.3), F(1,28)$ $=58.47, p<.001$, generalized eta squared $\left(\eta_{\mathrm{G}}^{2}\right)=0.336$. The Lure Type $\times$ Magnification interaction was significant, $F(1,28)=10.62, p<.001, \eta_{\mathrm{G}}^{2}=0.0292$, and post hoc $t$ tests $(p \mathrm{~s}<.001)$ indicated that the log slopes for the two types of lures differed for both magnification levels (see Fig. 4, left panel). This suggests that difference in log slopes between the two lure types was reduced but not eliminated when cortical magnification was compensated for.

When using the intercept coefficients as dependent variable instead, we found only a significant main effect of magnification: Intercepts were smaller with magnification $(472.1 \mathrm{~ms})$ than without $(506.6 \mathrm{~ms}), F(1,28)=43.48, p<.001, \eta_{\mathrm{G}}^{2}=$ 0.0744, (see Fig. 4, right panel).

Effects of target eccentricity The goal of this analysis was to examine how the effects observed above for magnification, lure type, and set size vary as a function of target eccentricity. A mixed-effects ANOVA was carried out on both logarithmic slopes and intercepts, using magnification, lure type, and target eccentricity $\left(4.5^{\circ}, 8.4^{\circ}\right.$, and $\left.15.4^{\circ}\right)$ as fixed-effect withinsubjects factors, and subject as a random effect. For these analyses we only report the effects that include the target eccentricity factor.

The ANOVA on logarithmic slopes showed a significant Eccentricity $\times$ Magnification interaction effect, $F(2,56)=$ $6.272, p<.005$, generalized eta squared $=0.0277$, indicating that log slopes increased with eccentricity when there was no magnification, but they did not vary with eccentricity when search items were magnified, $F(2,56)=8.25, p<.005$, generalized eta squared $=0.110$, and $F(2,56)=0.306, p=0.7$, respectively. In addition, this interaction was also qualified by lure type, $F(2,56)=3.370, p<.05$, generalized eta squared $=$ 0.011 . As seen in top left panel of Fig. 5 , the eccentricity effect under no magnification condition was larger for cyan circles than for orange diamonds.

The ANOVA on logarithmic intercepts indicated that log intercepts were significantly different at different levels of eccentricity, $F(2,56)=64.54, p<.001$, generalized eta squared $=$ 0.120 . It also revealed significant Eccentricity $\times$ Magnify

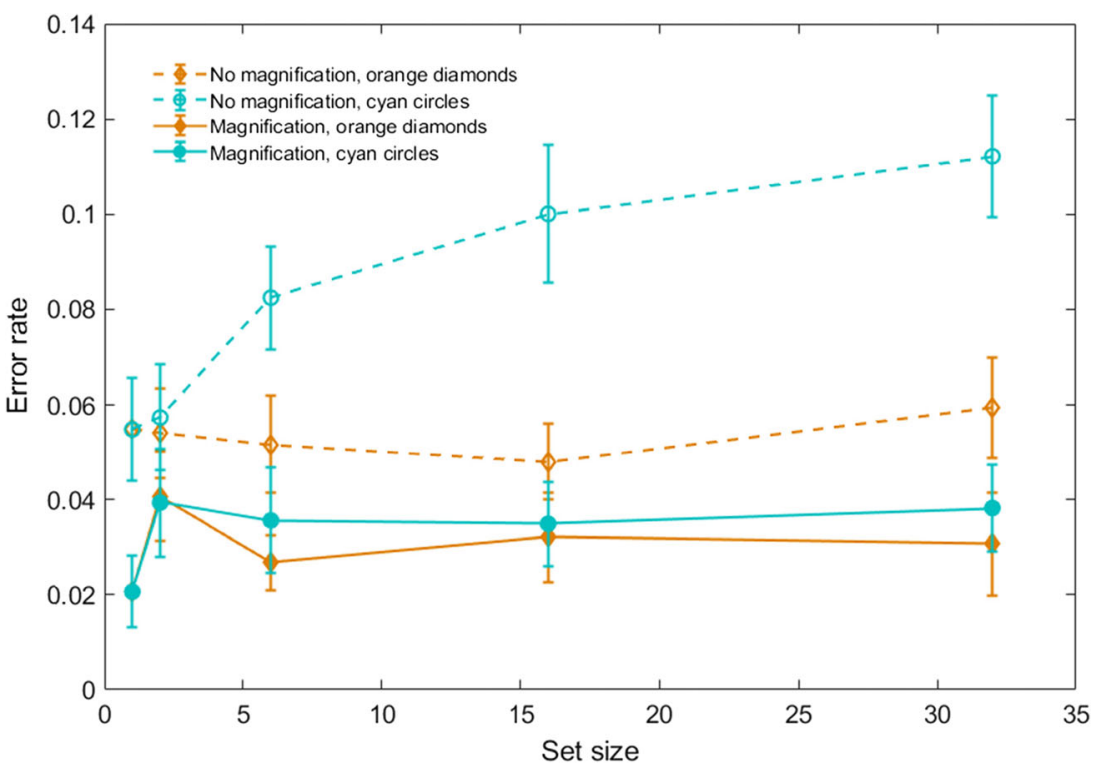

Fig. 3 Mean error rate as a function of set size, grouped by magnification and lure type. Error bars indicate one standard error of mean. (Color figure online) 

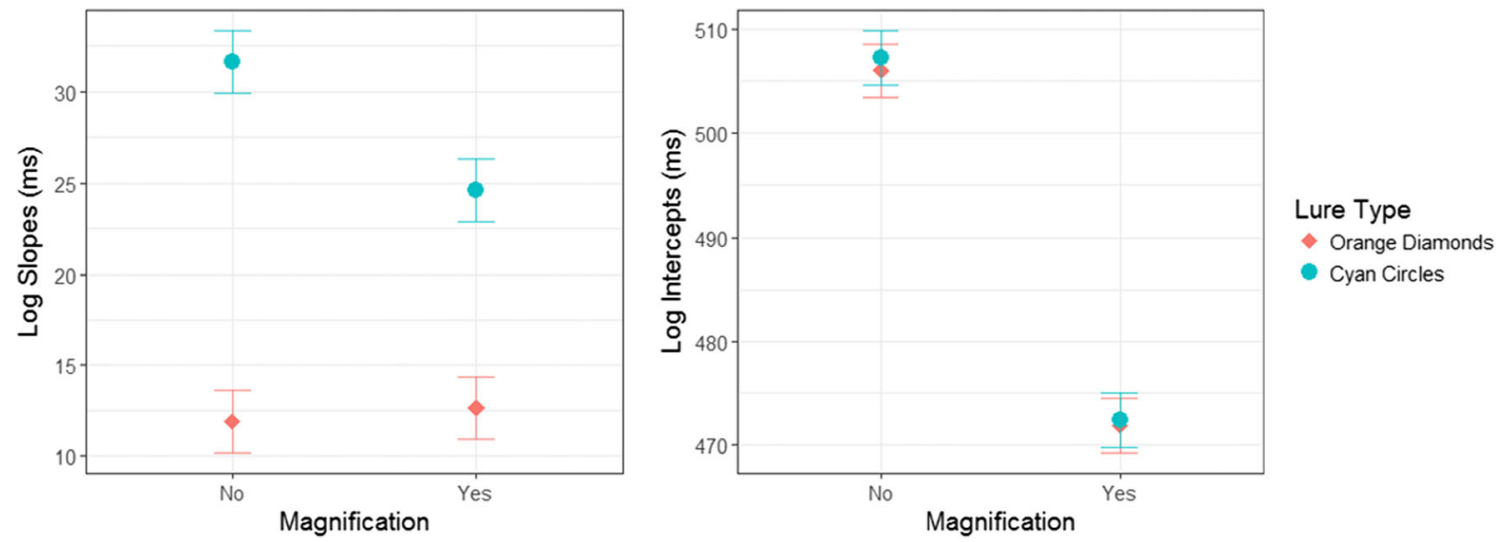

Fig. 4 Effects of lure type and magnification on estimated log slopes and intercepts of RT $\times$ Set Size functions. Error bars indicate Fisher's Least Significant Difference (LSD). (Color figure online)

interaction effects, $F(2,56)=46.85, p<.001$, generalized eta squared $=0.0619$, suggesting that effect of eccentricity on log intercepts are significantly different depending on whether or not cortical magnification was compensated for. Pair-wise $t$ tests indicated that when there was no magnification, log intercepts for target eccentricity of 15.4 degrees of visual angle differed significantly from the other two levels of eccentricities $(p<.001)$. In contrast, log intercepts at all target eccentricity levels did not differ from each other when there was cortical magnification compensation (all $p \mathrm{~s}>0.3$ ). This pattern of results is evident in Fig. 5, right column. There was also a small but significant Eccentricity $\times$ Lure Type interaction effect, $F(2,56)=3.696, p$ $<.05$, generalized eta squared $=0.0016$.

\section{Discussion}

Overall, these results provide clear evidence that RT $\times$ Set Size functions were better fitted by logarithmic functions compared with linear ones regardless of whether there was compensation for cortical magnification. Moreover, with or without magnification, slopes of these logarithmic functions were always modulated by target-lure similarity, with steeper slopes for more similar lures (cyan circles) and shallower ones for more dissimilar lures (orange diamonds). This is consistent with previous findings by Buetti et al. (2016) and Wang et al. (2017), suggesting that each item on the display meaningfully contributed to reaction time and that increasing target-lure
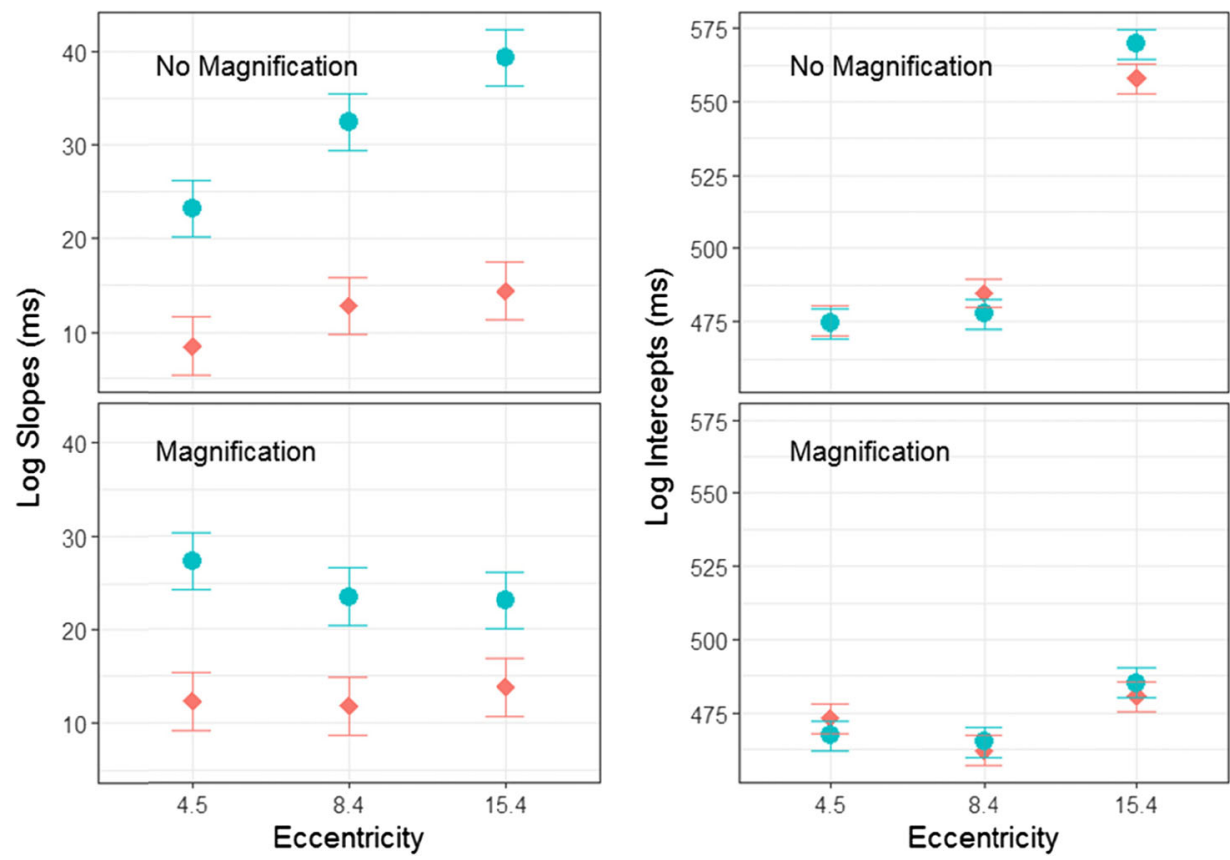

Lure Type

$\checkmark$ Orange Diamonds

- Cyan Circles

Fig. 5 Effects of target eccentricity and lure type on estimated log slopes (left column) and intercepts (right column) of RT $\times$ Set Size functions as a function of magnification. Error bars indicate Fisher's LSD. (Color figure online) 
similarity decreases processing efficiency of lure items. These results demonstrate that neither target eccentricity nor cortical magnification factors are responsible for the logarithmicity of the search functions observed in our previous studies. In addition, displays in the present study were designed to minimize crowding, which indicates that the logarithmic effects cannot simply be caused by crowding either, consistent with the results in Madison, Lleras, and Buetti (2017). In sum, the current findings are in support of our architecture-based account of logarithmic efficiency, as put forward in Buetti et al. (2016).

Although compensating for cortical magnification did not change the basic pattern of the log functions and the modulation of $\log$ slopes, we did find differences in the magnitude of various effects depending on whether search stimuli were magnified. Without compensation for cortical magnification, log slopes increased with target eccentricity; this effect disappeared with magnification-compensated displays. In Buetti et al.'s (2016) model of parallel evidence accumulation, the log slopes represent a parameter directly related to the time needed to evaluate the perceptual evidence and reach a decision as to whether the item is a lure. This finding suggests that it takes longer to evaluate a more eccentric item than an identical item that is closer to fixation, possibly due to having fewer neurons accumulating information about the more eccentric item. By magnifying the more eccentric item, roughly the same number of neurons are recruited to process the two items at different eccentricity, so that they are processed with similar efficiency. In addition, cortically magnifying stimuli also eliminated the effect of target eccentricity on log intercepts. Together, these findings suggest that our manipulation to compensate cortical magnification factor was effective. They also support Carrasco et al.'s $(1995,1998)$ proposal that most target eccentricity effects originate from low-level properties of the visual system (however, see Wolfe, O’Neill, \& Bennett, 1998, for a different view), so that appropriate changes in stimulus size can counteract eccentricity effects. Finally, the effect of lure type (or similarity) on logarithmic slopes was reduced but not eliminated when search displays contained magnified items. This reduction is evidence that covert attention effects can interact with eccentricity, as Carrasco and Frieder (1997) had pointed out.

By magnifying stimuli according to their eccentricity, the magnified displays have effectively a degree of heterogeneity in stimulus size. While stimuli heterogeneity was usually observed to hinder search performance (Duncan \& Humphreys, 1989), it was the opposite case in this experiment, similar to results of Carrasco and Frieder (1997) and Carrasco et al. (1998). RTs were faster for the magnified (thus heterogeneous) compared to the nonmagnified condition, as evidenced by the significant main effect of magnification on both log slopes and intercepts. Error rates were also visibly lower for the magnified condition (see Fig. 3). Several factors may have contibuted to this phenomenon. Magnifying stimuli in the periphery has a beneficial effect on processing efficiency by compensating for their neural representation. At the same time, because size is an irrelevant feature for the search task, the heterogeneity in this dimension could have a lower impact on search performance than relevant feature dimensions, according to the proposal of Müller, Heller, and Ziegler (1995). In addition, stimulus size was heterogeneous in a highly regular fashion, since they were magnified according to eccentricity. Little is known about whether the effect of feature heterogeneity depends on irregularity of that feature, so we may conjecture based on current data that regularity in a heterogeneous feature dimension could reduce the impact of heterogeneity on search efficiency. This possibility is supported by recent results in our lab, where heterogeneous search displays with spatially segregated (thus highly regular) distractors were searched more efficiently than randomly intermixed (thus irregular) displays.

\section{Conclusion}

The current study was designed to investigate the contribution of eccentricity (and cortical magnification) to search efficiency in fixed-target efficient search tasks. More specifically, we wanted to find out whether the logarithmic RT $\times$ Set Size functions observed in such tasks arise because of low-level visual factors or rather reflect intrinsic properties of a specific cognitive architecture. We provide evidence that cortical magnification differences do not change the qualitative nature of the search function: RT $\times$ Set Size functions were clearly more consistent with underlying logarithmic functions than linear ones under both magnified and nonmagnified conditions. The modulation of set size effects by lure type or target-lure similarity also remained significant after compensation for cortical magnification. Therefore, we conclude that the logarithmicity of RT $\times$ Set Size functions do reflect the existence of an underlying attentive parallel processing architecture that compares all items simultaneously to the target template and reaches separate decisions about each item in the display. Finally, consistent with previous findings in the literature, we confirmed that low-level visual factors like eccentricity and size have a direct impact on the efficiency of processing, likely reflecting differences in the accumulation speed of perceptual evidence that inform these decisions.

Acknowledgements The authors would like to thank Dr. Daniel Simons for his suggestion on data analysis approach. 


\section{References}

Buetti, S., Cronin, D. A., Madison, A. M., Wang, Z., \& Lleras, A. (2016). Towards a better understanding of parallel visual processing in human vision: Evidence for exhaustive analysis of visual information. Journal of Experimental Psychology: General, 145(6), 672.

Carrasco, M., Evert, D. L., Chang, I., \& Katz, S. M. (1995). The eccentricity effect: Target eccentricity affects performance on conjunction searches. Attention, Perception, \& Psychophysics, 57(8), 1241-1261.

Carrasco, M., \& Frieder, K. S. (1997). Cortical magnification neutralizes the eccentricity effect in visual search. Vision Research, 37(1), 63-82.

Carrasco, M., McLean, T. L., Katz, S. M., \& Frieder, K. S. (1998). Feature asymmetries in visual search: Effects of display duration, target eccentricity, orientation and spatial frequency. Vision Research, 38(3), 347-374.

Desimone, R., \& Duncan, J. (1995). Neural mechanisms of selective visual attention. Annual Review of Neuroscience, 18, 193-222.

Dow, B. M., Snyder, A. Z., Vautin, R. G., \& Bauer, R. (1981). Magnification factor and receptive field size in foveal striate cortex of the monkey. Experimental Brain Research, 44(2), 213-228.

Duncan, J., \& Humphreys, G. W. (1989). Visual search and stimulus similarity. Psychological Review, 96(3), 433.

Itti, L., \& Koch, C. (2001). Computational modelling of visual attention. Nature Reviews Neuroscience, 2, 194-203.

Kleiner, M., Brainard, D., Pelli, D., Ingling, A., Murray, R., \& Broussard, C. (2007). What's new in Psychtoolbox-3. Perception, 36(14), 1.

Lagroix, H. E., Di Lollo, V., \& Spalek, T. M. (2015). Is pop-out visual search attentive or preattentive? Yes!. Journal of Experimental Psychology: Human Perception and Performance, 41(2), 556.

Lleras, A., Buetti, S., \& Mordkoff, J. T. (2013). When do the effects of distractors provide a measure of distractibility. The Psychology of Learning and Motivation, 59, 261-316.

Madison, A., Lleras, A., \& Buetti, S. (2017). The role of crowding in parallel search: Peripheral pooling is not responsible for logarithmic efficiency in parallel search. Attention, Perception, \&
Psychophysics, 80(2), 353-372. https://doi.org/10.3758/s13414017-1441-3

Müller, H. J., Heller, D., \& Ziegler, J. (1995). Visual search for singleton feature targets within and across feature dimensions. Attention, Perception, \& Psychophysics, 57(1), 1-17.

Neider, M. B., \& Zelinsky, G. J. (2008). Exploring set size effects in scenes: Identifying the objects of search. Visual Cognition, 16(1), 1-10.

Pelli, D. G. (2008). Crowding: A cortical constraint on object recognition. Current Opinion in Neurobiology, 18(4), 445-451.

Rovamo, J., \& Virsu, V. (1979). An estimation and application of the human cortical magnification factor. Experimental Brain Research, 37(3), 495-510.

Strasburger, H., Rentschler, I., \& Jüttner, M. (2011). Peripheral vision and pattern recognition: A review. Journal of Vision, 11(5), 13-13. https://doi.org/10.1167/11.5.13

Theeuwes, J. (2010). Top-down and bottom-up control of visual selection. Acta Psychologica, 135, 77-99.

Townsend, J. T. (1990). Serial vs. parallel processing: Sometimes they look like Tweedledum and Tweedledee but they can (and should) be distinguished. Psychological Science, 1(1), 46-54.

Townsend, J. T., \& Ashby, F. G. (1983). Stochastic modeling of elementary psychological processes. Cambridge, UK: Cambridge University Press Archive.

Treisman, A. M., \& Gelade, G. (1980). A feature-integration theory of attention. Cognitive Psychology, 12(1), 97-136.

Wang, Z., Buetti, S., \& Lleras, A. (2017). Predicting search performance in heterogeneous visual search scenes with real-world objects. Collabra: Psychology, 3(1), 6. https://doi.org/10.1525/collabra.53

Wolfe, J. M. (1994). Guided Search 2.0: A revised model of visual search. Psychonomic Bulletin \& Review, 1, 202-238.

Wolfe, J. M., \& Horowitz, T. S. (2017). Five factors that guide attention in visual search. Nature Human Behaviour, 1. https://doi.org/10.1038/ s41562-017-0058

Wolfe, J. M., O’Neill, P., \& Bennett, S. C. (1998). Why are there eccentricity effects in visual search? Visual and attentional hypotheses. Perception \& Psychophysics, 60(1), 140-156. 